\title{
Analysis of Amphetamine Derivatives in Plasma Using Capillary Zone Electro- phoresis Coupled with Laser-Induced Fluorescence after Derivatization on Solid-Phase Extraction Support
}

\author{
Ludovic Gremaud§ab, Julie Schapplera ${ }^{\mathrm{a}}$, Jean-Luc Veuthey ${ }^{\mathrm{a}}$, Claude Rohrbasser ${ }^{\mathrm{b}}$, and \\ Serge Rudaz ${ }^{\star a}$ \\ §Dr. Max Lüthi Award Winner 2007
}

\begin{abstract}
Capillary electrophoresis (CE) is a very powerful analytical technique, consuming very small quantities of samples and solvents. Generally, a UV detector is coupled with CE but for the analysis of drugs in biological fluids, it is necessary to use a more sensitive and selective detector such as laser induced fluorescence (LIF). This detector was used to analyze various amphetamine derivatives which do not present native fluorescence and therefore a derivatization tag was employed to produce a fluorescent adduct. Fluorescamine, a fluorogene agent, was selected because it reacts with primary amines of amphetamines to give a fluorescent compound negatively charged, which can be analyzed by CZE-LIF. Taking into account the presence of many interfering compounds in biological fluids, analysis of these compounds requires a sample preparation prior to the analysis. Solid phase extraction (SPE) was selected and the derivatization step included. Analysis of independent plasma samples $(n=$ 6) exhibited a relative standard deviation (RSD) of $8 \%$. A relative recovery of $99 \%$ was observed for amphetamine, used as a model compound.
\end{abstract}

Keywords: Amphetamines · Capillary zone electrophoresis · Derivatization - Laser induced fluorescence . Solid phase extraction

${ }^{*}$ Correspondence: Dr S. Rudaz ${ }^{\mathrm{a}}$

Laboratory of Pharmaceutical Analytical Chemistry

School of Pharmaceutical Sciences - EPGL

University of Geneva, University of Lausanne

Bd d'Yvoy 20, 1211 Geneva 4

Tel.: $+41 / 22 / 379.65 .72$

Fax: $+41 / 22 / 379.68 .08$

E-Mail: serge.rudaz@pharm.unige.ch

aLaboratory of Pharmaceutical Analytical Chemistry

School of Pharmaceutical Sciences

University of Geneva, University of Lausanne

Boulevard d'Yvoy 20,

$\mathrm{CH}-1211$ Geneva 4,

bUniversity of Applied Sciences Western Switzerland College of Engineering and Architecture of Fribourg

Departement of Industrial Technology

Chemistry Section

Boulevard de Pérolles 80

$\mathrm{CH}-1700$ Fribourg

\section{Introduction}

Capillary electrophoresis (CE) is a powerful separation technique for analysis of a wide range of compounds in numerous analytical fields. It has several advantages, namely high efficiency, rapid method development, simple instrumentation and both low solvent and sample consumption. The most widely used detection technique in CE remains UV-VIS detection because of its on-capillary configuration, simplicity and quasi-universality at low wavelength. On the other hand, its sensitivity remains the major bottleneck, due to the small optical path length afforded by the capillary I.D. (in the $\mu \mathrm{m}$ range). Therefore, UV detection requires relatively high analyte concentration which is not compatible with bioanalytical applications. In fact, many drugs are present at low concentration levels due to their high distribution volumes. ${ }^{[1]}$ Appropriate sample preparation procedures for analyte preconcentration and/or highly sensitive detectors ${ }^{[2]}$ are mandatory. Other detection approaches such as mass spectrometry (MS) or fluorescence techniques should be considered. Indeed, the latter is generally very sensitive, since fluorescence signals are directly proportional to excitation power. Laser-induced fluorescence (LIF) is particularly adapted for CE because lasers are both easily focused and allow high efficient excitation. ${ }^{[3]}$ Furthermore, the high irradiance $(I)$ provided by a laser leads to better signal-to-noise ratio $(S / N)$, since signal intensity and noise are proportional to $I$ and $I^{1 / 2}$, respectively. Very low limits of detection (LOD) are provided by LIF detection with an improvement up to $10^{5}$ compared to conventional UV detection ${ }^{[4]}$ and LOD down to $10^{-12} \mathrm{M}$ have been reported.[5] To achieve such sensitivity levels, numerous conditions should be fulfilled. For non-fluorescent analytes and fluorophores that cannot be excited at the available laser wavelength, a chemical derivatization is required, ${ }^{[6-8]}$ leading to several drawbacks. For example, co-migrating interferences may be labeled 
providing fluorescence properties comparable to those of the analyte which increase the electropherogram complexity. ${ }^{[9,10]}$ In this paper, an analytical method for the analysis of psychostimulants in plasma by CZE after derivatization on solid phase extraction support was developed. This approach was applied to the analysis of amphetamine and derivatives.

\section{Materials and Methods}

\subsection{Chemicals}

Amphetamines and analogues were obtained from Lipomed AG (Switzerland). Derivative agents were provided by Molecular Probes (Oregon, USA) for CBQCA, Fluka (Buchs, Switzerland) for potassium cyanide, naphthalene-2,3-dicarboxaldehyde (NDA), fluorescamine and N,N-dimethylaminoethanthiol. Ultrapure water was supplied by a Milli-Q RG purification unit from Millipore (Bedford, MA, USA). N,N-dimethylformamide, formamide, acetonitrile, phosphoric acid, anhydride sulphate cupper, zinc sulphate, trichloroacetic acid, were provided by Fluka (Buchs, $\mathrm{CH}$ ). Tris (Trizma ${ }^{\circledR}$ base) was obtained from Riedel-de-Haën (Buchs, Switzerland). Methanol was obtained from Panreac (Spain). Ethanol was provided by Fisher Scientific (Leicestershire, UK) and boric acid, acetic acid and sodium hydroxide by Acros Organics (New Jersey, USA). Perchloric acid was obtained from Merck (Darmstadt, Germany). Blank plasma was obtained from the Blood Center of the Geneva Hospitals (Geneva, Switzerland).

\subsection{Background Electrolyte (BGE) and Sample Preparation}

The BGE consisted of a $25 \mathrm{mM}$ (ionic strength) borate buffer at $\mathrm{pH}$ 8.5. The $\mathrm{pH}$ value was measured with a SevenMulti $\mathrm{pH}$ meter (Mettler-Toledo, Schwerzenbach, Switzerland), calibrated daily with four aqueous solutions at $\mathrm{pH} 2.00,4.00,7.00$ and 10.00 from Riedel-de-Haën (Buchs, Switzerland). Stock standard solutions of analytes were prepared by dissolving analytes in $\mathrm{MeOH}$ to obtain a concentration of $1 \mathrm{mg} \cdot \mathrm{ml}^{-1}$. These stock standard solutions were stored at $4{ }^{\circ} \mathrm{C}$ until use. Standard solutions of analytes at the desired concentrations were prepared daily by diluting stock solutions in water. Sample solutions were stable for more than two days at $4{ }^{\circ} \mathrm{C}$ and no degradation occurred for the tested analytes during analysis.

\subsection{Plasma Sample}

Plasma were obtained from blood samples by centrifugation and stored at -20 ${ }^{\circ} \mathrm{C}$. Plasma samples containing different amounts of amphetamine analogues and internal standard (I.S.) were prepared by spiking the plasma with a known amount of racemic amphetamine analogues and I.S. before the protein precipitation (PP). For this purpose, $200 \mu \mathrm{l}$ of ACN was added to $100 \mu$ l of spiked plasma. After vortexmixing for $30 \mathrm{~s}$, the sample was centrifuged for $5 \mathrm{~min}$ at $3100 \mathrm{~g}$. $200 \mu \mathrm{l}$ of the supernatant was transferred for derivatization (see Section 2.4).

\subsection{Derivatization Procedure}

For liquid derivatization, $70 \mu \mathrm{l}$ of amphetamines solution, $60 \mu$ l of fluorescamine solution at $1000 \mathrm{ppm}$ and $200 \mu \mathrm{l}$ of borate buffer at $25 \mathrm{mM}, \mathrm{pH} 9.2$ was added to 840 $\mu l$ of buffer. The mixture was stirred, let at room temperature for $5 \mathrm{~min}$ and injected.

For SPE derivatization, an Oasis ${ }^{\circledR}$ HLB column (Waters, Millford, USA) was employed. $200 \mu \mathrm{l}$ of the supernatant diluted with $800 \mu \mathrm{l}$ of borate buffer solution $25 \mathrm{mM}$ at $\mathrm{pH} 8.5(1: 4 \mathrm{v} / \mathrm{v})$, was loaded on the extraction support. The support was washed with $750 \mu$ l of borate buffer. 300 $\mu l$ of $1000 \mathrm{ppm}$ fluorescamine was percolated throughout the cartridge. Finally, 300 $\mu \mathrm{l}$ of $\mathrm{MeOH}$ was used for the elution and the solution directly injected as indicated in Section 2.5.1.

\subsection{Instrumentation \\ 2.5.1. Capillary Electrophoresis}

CE experiments were performed with an HP 3DCE system (Agilent, Waldbronn, Germany) equipped with on-capillary diode array detector, autosampler and power supply able to deliver up to $30 \mathrm{kV}$. Separation was performed in an uncoated fused silica (FS) capillary (BGB Analytik AG, Böckten, Switzerland) with a $50 \mu \mathrm{m}$ I.D., total length of $47 \mathrm{~cm}$ and effective length of $33.5 \mathrm{~cm}$ for LIF detection. Experiments were carried out in positive polarity mode, with the anode at the inlet and the cathode at the outlet. A constant voltage of $30 \mathrm{kV}$, with an initial ramping of $1000 \mathrm{~V} \cdot \mathrm{s}^{-1}(30$ s), was applied during analysis, and the capillary temperature was maintained at $30^{\circ} \mathrm{C}$. Samples were kept at ambient temperature in the autosampler and injected in hydrodynamic mode at 40 mbar for 10 s (equivalent to $2.2 \%$ of capillary length). Before its first use, the capillary was sequentially rinsed with $\mathrm{MeOH}, \mathrm{NaOH}(0.1$ $\mathrm{M})$, water and fresh BGE for 5 min each. Prior to each sample injection, the capillary was rinsed at 2 bar for 1 min with fresh BGE. After each run, the capillary was rinsed at 1 bar with $\mathrm{NaOH}(0.1 \mathrm{M})$ and fresh BGE for $1 \mathrm{~min}$. A voltage of 30 $\mathrm{kV}$ was applied for $1 \mathrm{~min}$ to refresh the capillary wall and obtain repeatable electroosmotic flow. ${ }^{[11]}$ When not in use, the capillary was rinsed with water and then dry stored. As the electrophoretic process alters the running buffer $\mathrm{pH}$ by electrolysis and subsequently changes migration times, the separation buffer was refreshed every four runs.

\subsubsection{Laser Induced Fluorescence}

LIF detection was performed with a ZetaLIF Evolution system (Picometrics, Ramonville, France) hyphenated to the CE system. A diode laser providing fluorescence excitation at $410 \mathrm{~nm}$ was selected and coupled to the detector with an optical fiber. The laser produced $15 \mathrm{~mW}$ of average power operated in continuous wave mode. A photomultiplier detector was used to measure fluorescence intensity.

\subsubsection{Software}

Buffer and BGE solutions were prepared with the help of PHoEBuS software version 1.3 (Analis, Namur, Belgium). CE ChemStation (Agilent, Waldbronn, Germany) was used for CE instrument control and Azur version 4.0 (Datalys, Saint Martin d'Hères, France) was used for data acquisition, data handling and analytical parameter calculation (efficiency, S/N).

\section{Results and Discussion}

\subsection{Choice of Analytes}

Amphetamine and its analogues are drugs widely consumed in Europe in 'rave parties' or as doping agents in sport. These compounds have a potent central nervous system stimulating effect. Numerous amphetamine analogues could be used including amphetamine (AMP), methylenedioxyamphetamine (MDA), 4-methylthioamphetamine (MTA), 3,4-methylenedioxyphenylbutanamine (BDB), mescaline $(3 \mathrm{CH})$ and 4-bromo-2,5-dimethoxyphenylethylamine (2CB). Chemical structures are reported in Fig.1.

A large number of studies has been published on amphetamine and its derivatives, with LC-UV or GC-FID as analytical methods and detection limits (LOD) around $10^{-6}-10^{-8} \mathrm{M}$ were reported. As indicated elsewhere, the use of LIF detection could reduce the LOD to around $10^{-12}-10^{-16}$ M. ${ }^{[12]}$ This could be of utmost interest when dealing with biological fluids such as plasma where psychostimulants are widely distributed in the body and consequently present at low concentrations. For these chemical structures which do not possess a strong fluorophore, a derivatization procedure should be achieved with a suitable fluorescent tag, matching the laser excitation wavelength.

\subsection{Derivatization Conditions and Sample Preparation}

In order to employ capillary zone electrophoresis (CZE), the presence of a charge on the final complex is mandatory. This technique limits many problems such as fluorescence quenching by the presence 


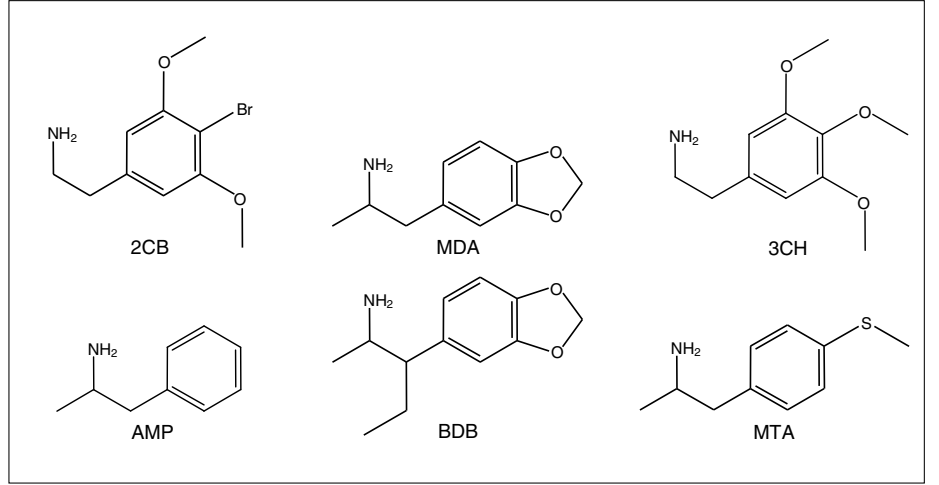

Fig. 1. Chemical structures of tested amphetamine derivatives. Amphetamine (AMP), methylenedioxyamphetamine (MDA), 4-methylthioamphetamine (MTA), 3,4-methylenedioxyphenylbutanamine (BDB), mescaline $(3 \mathrm{CH})$ and 4-bromo2,5-dimethoxyphenylethylamine $(2 \mathrm{CB})$

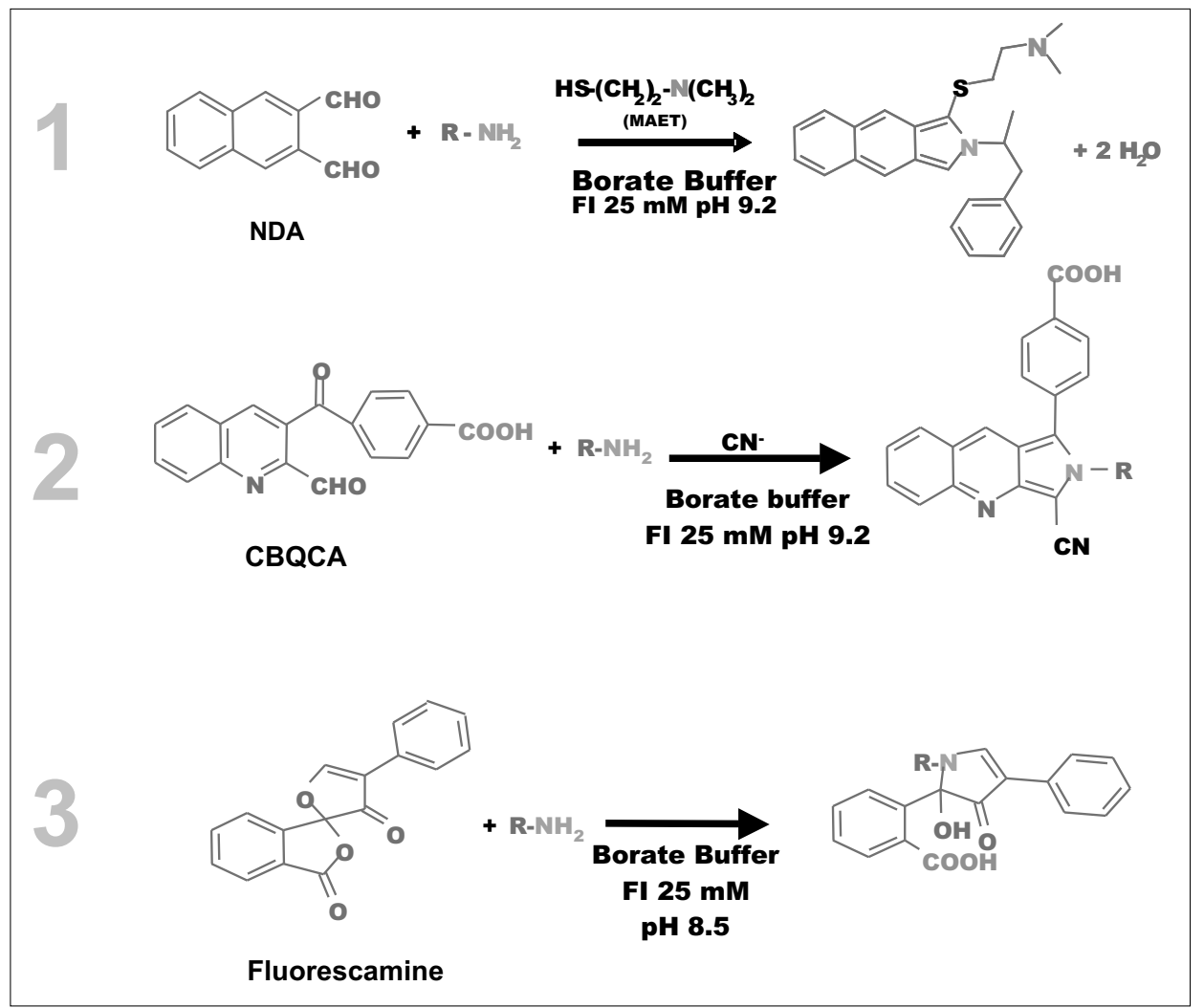

Fig. 2. Tested reactions for amphetamine derivation. For experimental conditions, see text.

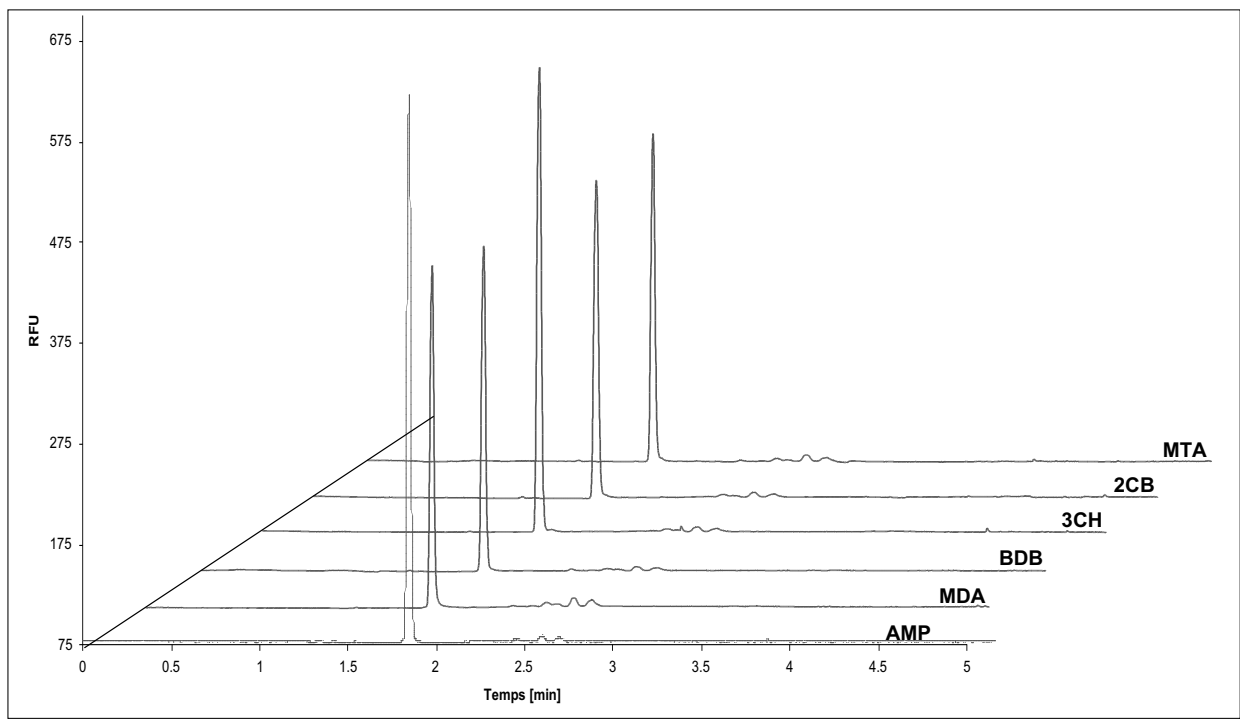

Fig. 3. Electropherograms of amphetamine derivatives at $10 \mathrm{ppm}$ separated by CZE-LIF; Capillary: tot. length $47.0 \mathrm{~cm}$, eff. length $33.5 \mathrm{~cm}$, I.D. $50 \mu \mathrm{m}$; temperature: $25^{\circ} \mathrm{C}$; BGE: $\mathrm{Fl} 25 \mathrm{mM}$ borate buffer pH 8.5; hydrodynamic injection $10 \mathrm{~s} 40$ mbar, $2.2 \%$. eff. vol.; voltage: $30 \mathrm{kV}$, ramp $30 \mathrm{~s}$; detection: LIF $410 \mathrm{~nm}, 15 \mathrm{~mW}$, rt $0.5 \mathrm{~s}$, PMT $570 \mathrm{~V}$ of surfactant agent or bubble formation in the capillary when micellar electrokinetic chromatography (MECK) is employed for separating neutral compounds. Therefore, several derivatization fluorescent tags, producing compounds at $410 \mathrm{~nm}$ and containing an ionizable function were studied (Fig. 2).

As example, NDA which provides high complex stability and good sensitivity at $442 \mathrm{~nm}$, especially for primary amines, could not be used as a fluorescent probe. The reaction with amphetamines performed in the dark and in presence of $\mathrm{CN}-$ (which acts as a nucleophilic agent), formed neutral complexes which cannot be separated by CZE. The addition of N,Ndimethylaminoethanthiol (MAET) during the reaction (Fig. 2, reaction 1) to obtain compounds which can be protonated in acidic media was tested without success. In fact, isoindole compounds were probably instable and no fluorescence was observed. ${ }^{[13-17]}$ Another procedure following the same methodology was used for the derivatization. The use of a nucleophilic probe, CBQCA was considered (Fig. 2, reaction 2). This reaction, well known in organic synthesis, is not often used in analytical chemistry. ${ }^{[18-20]}$ CBQCA is a probe quite similar to NDA but gives derivatized compounds that can be negatively charged at appropriate $\mathrm{pH}$ thanks to the presence of a carboxylic function. With this probe, a low fluorescence signal was observed, attributed to a non-optimal excitation wavelength. In fact, for this tag a $480 \mathrm{~nm}$ laser would have been more appropriate. Furthermore, this tag was not considered for economical reasons.

Finally, the last probe used for derivatization of non-fluorescent amphetamine analogues was fluorescamine (Fluram). ${ }^{[21,22]}$ This probe reacted with primary amines, giving compounds fluorescent at $410 \mathrm{~nm}$ (see Fig. 2, reaction 3). Thanks to a structural rearrangement, a carboxylate function was induced. This function allowed the migration of the derivatized amphetamines as anions using a BGE at relatively high $\mathrm{pH}$ (borate buffer, $\mathrm{pH}$ 8.5). The derivatization product was found stable for more than 90 min with a reaction time of about $5 \mathrm{~min}$, which was particularly interesting compared to CBQCA where the reaction took 90-120 min. The derivatization method with Fluram was optimised on amphetamine analogues in aqueous sample spiked at $10 \mathrm{ppm}$. As presented in Fig. 3, all compounds of interest were found to be detected by CE-LIF.

In order to adapt this derivatization procedure to plasma, a protein precipitation (PP) was carried out to eliminate potential endogenous interfering compounds. Plasma samples were spiked at $1 \mathrm{ppm}$ with amphetamine analogues and different PP 


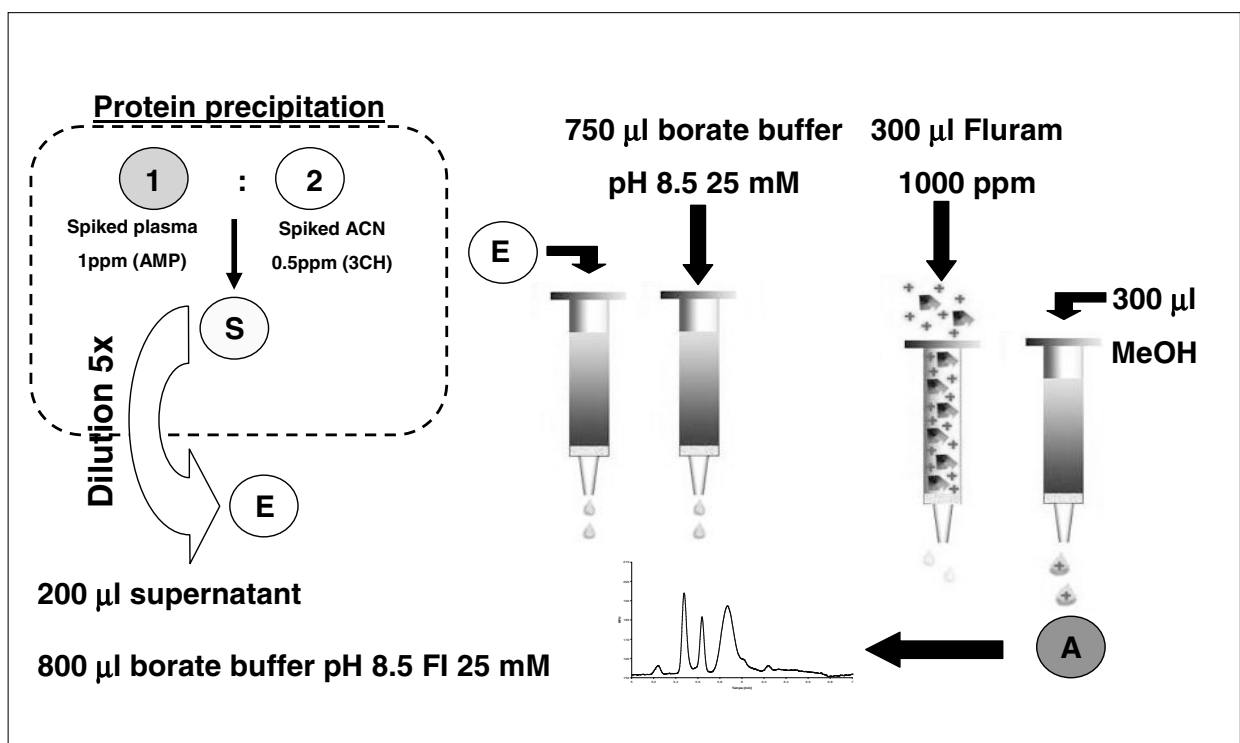

Fig. 4. Sample preparation methodology for amphetamine derivatization on solid phase extraction support after protein precipitation of plasma

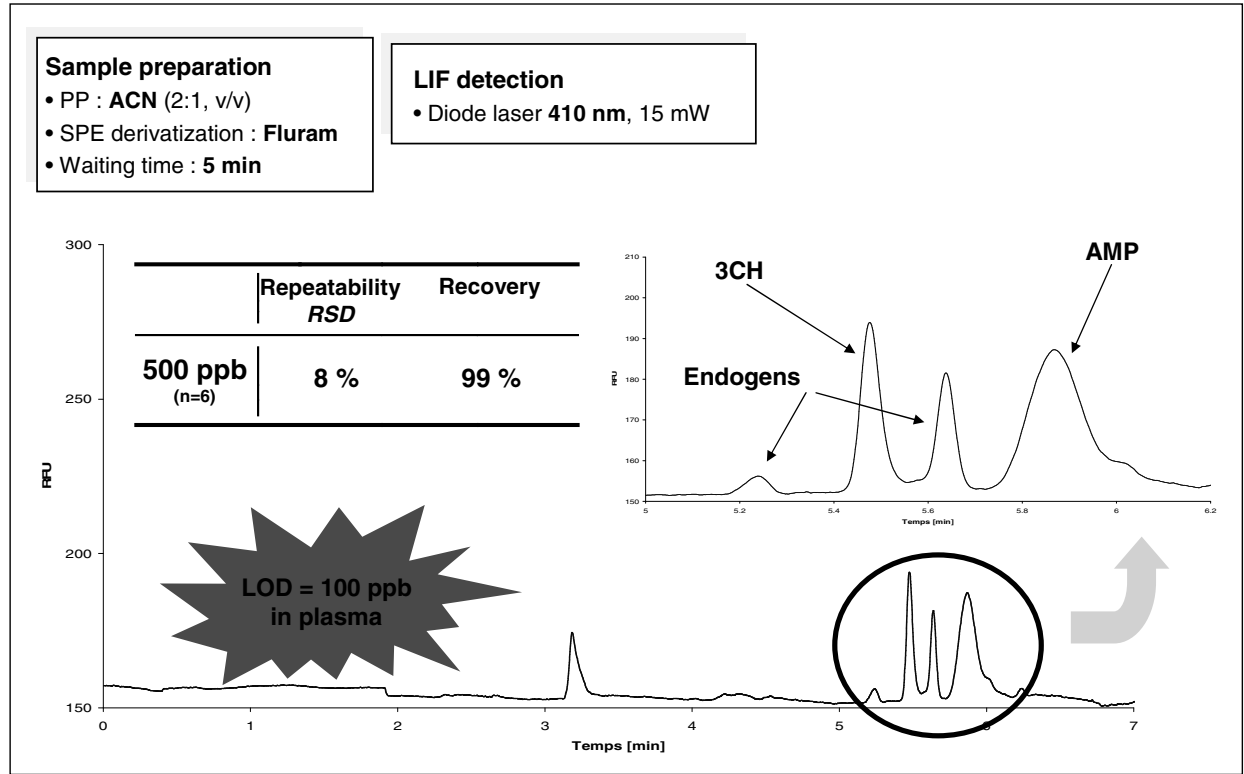

Fig. 5. Obtained electropherogram for the analysis of amphetamine (AMP) as model compound and mescaline $(3 \mathrm{CH})$ as I.S.

procedures tested. The nature of precipitation agent and various ratio of plasma/ precipitation agent were evaluated. Acetonitrile (ACN), 20\% trichloroacetic acid (TCA), 5\% perchloric acid (PA), zinc sulphate $\left(\mathrm{Zn}_{\mathrm{S}} \mathrm{O}_{4}\right)$ and copper sulphate $\left(\mathrm{CuSO}_{4}\right)$ in $1: 1,2: 1$ and $4: 1,(\mathrm{v} / \mathrm{v})$ ratios were employed. The derivatization with Fluram was tested on each supernatant and the method selectivity assessed by visual inspection of the obtained electropherograms. Three reagents were found to provide satisfactory results for PP: PA, TCA and ACN. The latter was estimated to give the cleanest supernatant and the derivatization with Fluram on precipitated plasma samples gave similar performance to aqueous standard.
The next step was to transfer the derivatization procedure to solid phase extraction support. For this purpose, the affinity of amphetamine compounds was tested on different solid phase chemistries mainly based on hydrophobic interactions (HLB, C18). It should be emphasized that both supports retained all the tested analytes following the basic manufacturer's recommendations. Oasis HLB, a hydrophile-lipophile balanced copolymer, was finally selected on recovery based results (data not shown). As $\mathrm{PP}$ with $\mathrm{ACN}$ involved the final presence of an important volume of organic solvent in the sample to be deposited on the cartridge, it was necessary to dilute the sample before SPE to avoid analytes loss during the loading step. A five-fold dilution was achieved with the separation buffer ( $\mathrm{pH} 8.5)$ to increase the hydrophobic retention of the cationic analytes. The Fluram solution was then percolated to derivatize the deposited analyte directly on the solid surface. Pure $\mathrm{MeOH}$ completely eluted the compounds and was directly injected in the CE-LIF system. Fig. 4 shows the optimized procedure of sample preparation and SPE derivatization mode.[23,24]

\subsection{Application to Biological Samples}

Amphetamine (AMP) was finally selected as a model analyte with mescaline $(3 \mathrm{CH})$ as internal standard (I.S.) to evaluate the quantitative performance of the analytical procedure. As presented in Fig. 5, analysis of spiked plasma sample with both compounds was performed including sample preparation (PP) and derivatization procedure on SPE. The presence of endogenous components migrating between $3 \mathrm{CH}$ and AMP was observed without any interfering effect. The detection limit of amphetamine in plasma (LOD), expressed as the signal to noise ratio $(S / N)$ of 3 , was found to be at $100 \mathrm{ng} / \mathrm{ml}$. Quantification limit (LOQ) was estimated at $500 \mathrm{ng} / \mathrm{ml}(S / N>10)$. Relative standard deviation (RSD) values on peak areas of plasma samples spiked with $500 \mathrm{ng} / \mathrm{ml}$ of amphetamine analogues were lower than $10 \%(8.0 \% ; n=6)$. The mean recovery at $500 \mathrm{ng} / \mathrm{ml}$ was excellent, with a process efficiency of $99 \%(n=6)$. These values reflect the entire process of the method developed: yield of reaction, derivatization on SPE, precipitation of proteins and analysis.

\section{Conclusion}

A CZE-LIF method for analyzing amphetamine compounds in plasma samples after derivatization on solid phase extraction support was developed. LIF detection is very sensitive and thus allows detection of very low concentrations of amphetamines, widely distributed in the body. Because amphetamines are not fluorescent, a derivatization procedure was developed. Fluorescamine was chosen as derivative agent for several reasons, among them, complexes presented an ionizable function, compatible with a CZE separation. The analysis of these compounds in body fluids involved a sample preparation prior to analysis in order to increase method selectivity and sensitivity. Spiked plasma samples were extracted on solid support (SPE) and analytes of interest derivatized on the cartridge. The compounds were separated by CZE and finally detected by LIF at $410 \mathrm{~nm}$. The quantitative performance of the CE-LIF 410 method developed in this work was evaluated in terms of selectivity, recovery, precision (repeat- 
ability), limit of detection (LOD) and limit of quantitation (LOQ).

\section{Acknowledgements}

We would like to acknowledge the SSC for the Max Lüthi Award, EIA-FR for his support and all collaborators of Pharmaceutical Analytical Chemistry of University of Geneva. Dr. N. Lange is warmly acknowledged for his stimulating discussion concerning the tag choice.

Received: March 7, 2008

[1] C. M. Boone, J. C. Waterval, H. Lingeman, K. Ensing, W. J. Underberg, J. Pharm. Biomed. Chem. 1999, 20,831.

[2] G. Hempel, Electrophoresis 2000, 21, 691.

[3] M. E. Johnson, J. P. Landers, Electrophoresis 2004, 25, 3513.

[4] B. M. Simonet, A. Rios, M. Valcarcel, TrAC 2003, 22, 605.

[5] M. Albin, P. D. Grossman, S. E. Moring, Anal. Chem. 1993, 65, 489A.

[6] J. C. Waterval, H. Lingeman, A. Bult, W. J. M. Underberg, Electrophoresis 2000, 21, 4029.

[7] W. J. M. Underberg, J. C. Waterval, Electrophoresis 2002, 23, 3922.

[8] T. Fukushima, M. Usui, T. Santa, K. Imai, J. Pharm. Biomed. Chem. 2003, 30, 1655.

[9] A. J. G. Mank, H. Lingemann, C. Gooijer, $\operatorname{TrAC}$ 1996, 15, 1 .

[10] G. Kavran-Belin, S. Rudaz, J. L. Veuthey, J. Sep. Sci. 2005, 28, 2187.

[11] M. M. Hsieh, Y. C Kuo, M. J. Lyu, H. T. Chang, J. Chromatogr. A 2000, 898, 133.

[12] N. Kuroda, R. Nomura, O. Al-Dirbashi, S. Akiyama, K. Nkashima, J. Chromatogr. A 1998, 798, 325 .
[13] R. G. Carlson, K. Srinivasachar, R. S Givens, B. K. Matuszewski, J. Org. Chem. 1986, 51, 3978.

[14] J. F. Stobaugh, A. J. Repta, L. A. Sternson, J. Org. Chem. 1984, 49, 4306.

[15] S. Stoney, D. F. Johnson, J. Org. Chem. 1978, 43, 2886.

[16] C.L.Copper, G.E.Collins, Electrophoresis 2004, 25, 897

[17] M. Lacroix, J.-C. Garrigues, P. Naccache, V. Poinsot, F. Couderc, 'A new strategy to selectively quantitate neuroactive amines in mass spectrometry and laser-induced fluorescence', The 26th International Symposium on Chromatography Copenhagen, $\mathrm{Pb} 04,2006$.

[18] J. Liu, O. Shirota, D. Wiesler, M. Novotny, Proc. Natl. Acad. Sci USA 1991, 88, 2302.

[19] M. Lacroix, V. Poinsot, C. Fournier, F. Couderc, Electrophoresis 2005, 26, 2608.

[20] Molecular Probes, ATTO-TAG CBQCA and ATTO-TAG FQ, https://catalog. invitrogen.com/index.cfm?fuseaction $=$ viewCatalog. viewProductDetails \&prod uctDescription $=6780 \&$ catname $=$ Europe an\%20Main, 1-4, 2001.

[21] M. Albin, R. Weinberger, E. Sapp, S Moring, Anal. Chem. 1991, 63, 417.

[22] E. L. Gump, C. A. Monnig, J. Chromatogr. A 1995, 715, 167.

[23] P. Drew, J. A. Lapos, A. D. Jones, A. G. Ewing, Analytical Biochemistry 2003, 22, 68.

[24] C. Li, Z. Chen, D. Wen, J. Zhang, W. Cong, B. Yu, Y. Liao, H. Liu, Electrophoresis 2006, 27, 2152. 